\title{
False alarm for a cardiac pseudomass during a pulmonary vein isolation for paroxysmal atrial fibrillation
}

\author{
Sofia Chatzikyriakou*, Daniela Mirica, Antoine Bondue and Ruben Casado Arroyo \\ Department of Cardiology, CUB-Hôpital Erasme, Université Libre de Bruxelles, Route de Lennik 808, 1070 Brussels
}

\begin{abstract}
Incidental findings during imaging studies may constitute a diagnostic pitfall. We report a case of a cardiac mass in right atrium identified during a pulmonary vein isolation for paroxysmal atrial fibrillation. A physiological tissue corresponding to a prominent crista terminalis was assumed and confirmed by multimodality imaging techniques, while a malignancy was, also, ruled out concerning the personal history of the patient. A prominent crista terminalis is frequent finding in magnetic resonance, but its appearance prevalence in transthoracic echocardiography is unknown. A proper diagnosis of a prominent crista terminalis may be facilitated in the future by the improvement of ultrasound imaging techniques and the awareness of its existence.
\end{abstract}

\section{Case report}

An 86 years old woman with a history of paroxysmal and symptomatic (EHRA IIb) atrial fibrillation (AF), refractory to antiarrhythmic drugs (AAD) was admitted to our hospital for a pulmonary veins isolation (PVI) by radiofrequency ablation [1]. Her medical history included arterial hypertension, hypercholesterolemia and a colectomy for colorectal polyps fifteen years ago. The patient presented repetitive episodes of pre-syncope and palpitations four months ago, where a paroxysmal AF was diagnosed, a transthoracic echocardiogram revealed normal left ventricular ejection fraction, no significant valvular disease, normal structures and diastolic dysfunction grade II, and a medical treatment of an anticoagulant, AAD IC and b-blocker was initiated. Despite the medical treatment, the patient remained symptomatic and an interventional treatment approach was decided. The PVI was performed under general anesthesia and a transesophageal echocardiography (TEE) was performed to confirm a left atrial appendage free of thrombi and to guide the trans-septal puncture. However, the TEE revealed an echogenic, round shaped, broad based mass, attached but not infiltrative to the posterolateral wall of the right atrium, with smooth surface, with synchronous motion with the wall and without any calcifications. (Figures 1 and 2, Supplemental Digital Content Videos 1 and 2). We assumed the presence of a prominent crista terminalis and the patient underwent successful PVI without any complications and without being jeopardized by the prominent crista terminalis (Figure 3). The diagnosis of a prominent crista terminalis was, also, confirmed by a cardiac MRI (Figure 4 ) and a PET computed tomography was performed, concerning the colectomy in the past, which ruled out any malignancy. The patient remains free of symptoms 6 months post procedure.

\section{Discussion}

Crista terminalis is a fibromuscular ridge formed by the junction of the sinus venosus and the primitive right atrium. The prevalence of a crista terminalis prominence and its correlation with atrial arrhythmias are not well known [2]. However, a prominent crista terminalis identification is mandatory in every day clinical practice

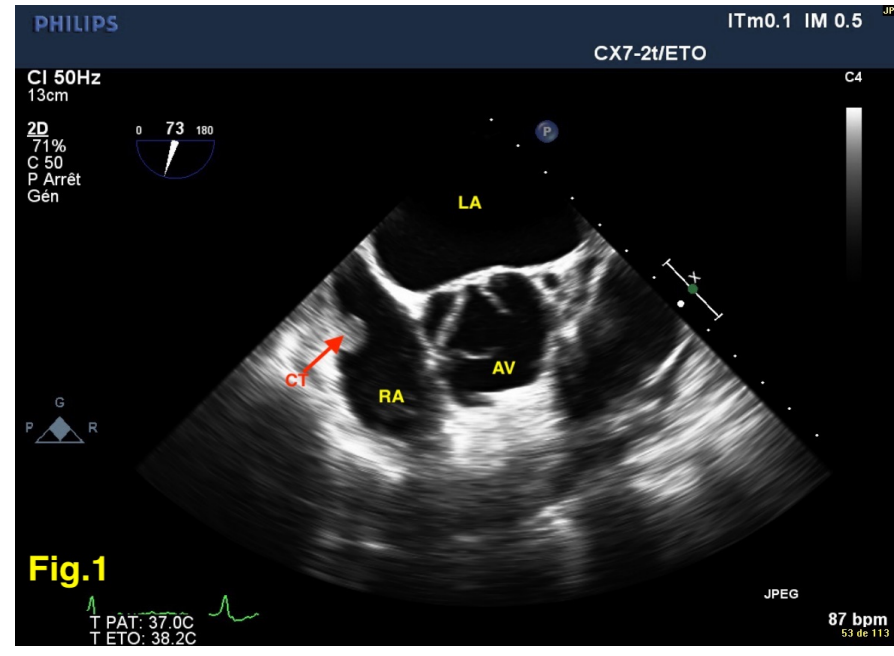

Figure 1. Transesophageal echocardiography at the level of the aortic valve CT: Crista Terminalis, RA: Right Atrium, LA: Left Atrium, AV: Aortic Valve

and specially during electrophysiology studies and ablations, where a manipulation of catheters in the right atrium presents a routine phenomenon. Finally, a proper diagnosis of a prominent crista terminalis can be facilitated by an increasing suspicion threshold of its existence and by the transesophageal echocardiography and the magnetic resonance, but rarely by the transthoracic echocardiography $[3,4]$ and there is no need for follow up imaging studies.

${ }^{\star}$ Correspondence to: Sofia Chatzikyriakou, Department of Cardiology, CUBHôpital Erasme, Université Libre de Bruxelles, Route de Lennik 808, 1070 Brussels, Tel: 0032(0)25553907; E-mail: sofia.chatzikyriakou@erasme.ulb.ac.be

Key words: cardiac pseudomass, paroxysmal atrial fibrillation, echocardiography Received: February 20, 2019; Accepted: February 28, 2019; Published: March 07,2019 


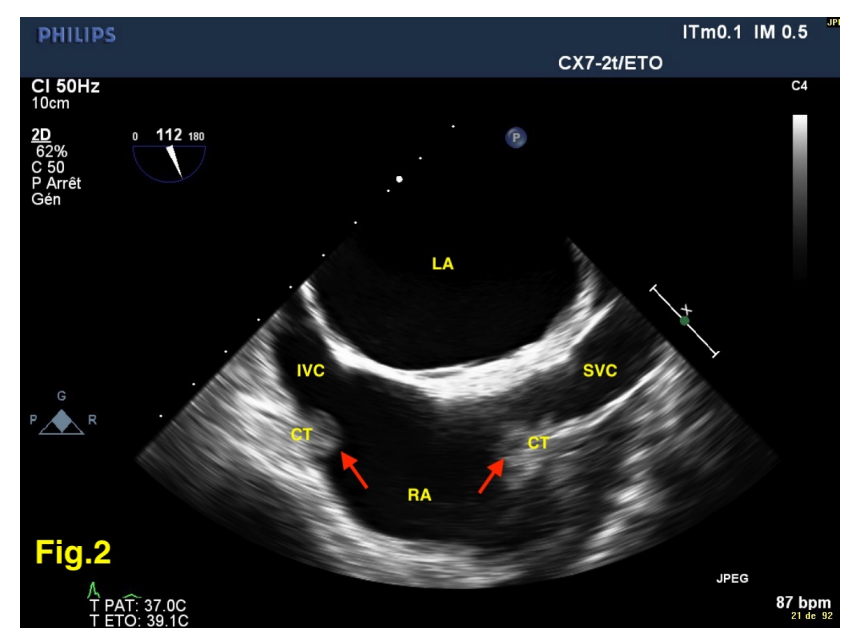

Figure 2. Transesophageal echocardiography: bicaval view

CT: Crista Terminalis, RA: Right Atrium, LA: Left Atrium, IVC: Inferior Vena Cava, SVC: Superior Vena Cava.

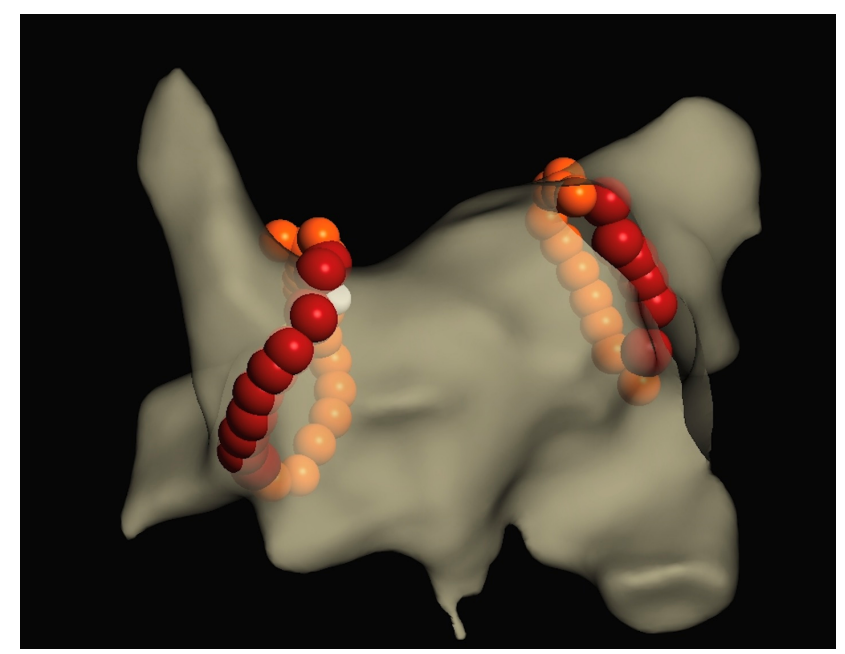

Figure 3. Radiofrequency point by point circumferential pulmonary veins isolation

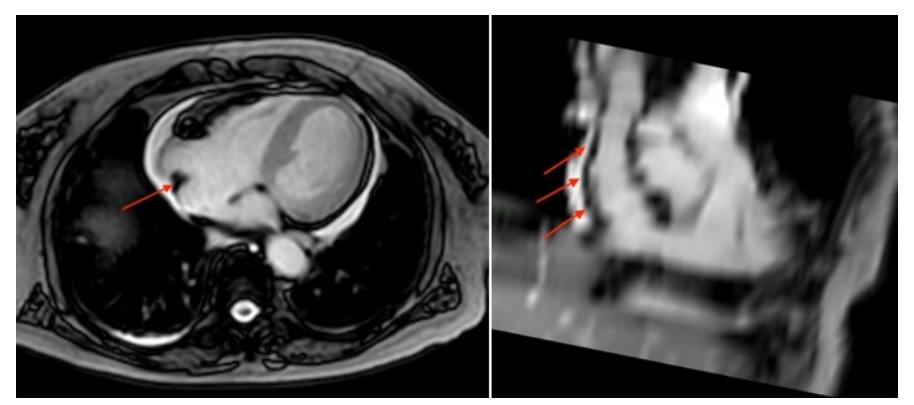

Figure 4. Magnetic resonance images. Right panel: axial view, Left panel cranio-caudal view. Arrows indicate the crista terminalis

\section{Consent}

The authors confirm that written consent for submission and publication of the images and associated text has been obtained from the patient

\section{Conflict of interest}

No conflict of interest, financial or otherwise concerning for all the authors.

\section{Acknowledgements}

The authors would like to acknowledge the contribution of the radiologist who acquired the MRI images, Dr Thoma Philippe.

\section{References}

1. Kirchhof P, Benussi S, Kotecha D, Ahlsson A, Atar D, et al (2016) 2016 ESC Guidelines for the management of atrial fibrillation developed in collaboration with EACTS. Eur Heart J 37: 2893-2962. [Crossref]

2. Akcay M, Bilen ES, Bilge M, Durmaz T, Kurt M (2007) Prominent crista terminalis: as an anatomic structure leading to atrial arrhythmias and mimicking right atrial mass. $J$ Am Soc Echocardiogr 20: 9-10. [Crossref]

3. D'Amato N, Pierfelice O, D'Agostino C (2009) Crista terminalis bridge: a rare variant mimicking right atrial mass. Eur J Echocardiogr 10: 444-445. [Crossref]

4. Pharr JR, West MB, Kusumoto FM, Figueredo VM (2002) Prominent crista terminalis appearing as a right atrial mass on transthoracic echocardiogram. $J \mathrm{Am} \mathrm{Soc}$ Echocardiogr 15: 753-755. [Crossref]

Copyright: (C2019 Chatzikyriakou S. This is an open-access article distributed under the terms of the Creative Commons Attribution License, which permits unrestricted use, distribution, and reproduction in any medium, provided the original author and source are credited. 